\title{
Bulk Properties of Syndiotactic 1,2-Polybutadiene. I. Thermal and Viscoelastic Properties
}

\author{
Yutaka Oвata, Chikao Tosaki, and Masaru Ikeyama \\ Research Laboratory, Japan Synthetic Rubber Co., \\ 7569, Ikuta, Tama-ku, Kawasaki, Japan.
}

(Received July 5, 1974)

\begin{abstract}
In order to find some of the fundamental characteristics of 1,2-polybutadiene, a series of samples with different degrees of crystallinity $\chi$ (below 46\%) were prepared. Infrared and ${ }^{13} \mathrm{C}-\mathrm{NMR}$ spectra indicated that the weight fraction of the 1,2 unit with a syndiotactic configuration is mainly responsible for $\chi$. X-ray diffraction analysis, differential scanning calorimetry (DSC) analysis and dynamic measurement of viscoelasticity showed the following: (1) The structure of the unit cell of the crystal is identical with that assigned by G. Natta to syndiotactic 1,2-polybutadiene (orthorhombic). (2) The fusion of the crystal occurs over a wide range of temperature and $\chi$ decreases almost linearly with increasing temperature. (3) Certain characteristic temperatures of the fusion behavior of the crystal increases linearly with $\chi$. (4) The specific heat of fusion is proportional of $\chi$ and an extrapolation gives $14.5 \mathrm{cal} / \mathrm{g}$ for $100 \%$ crystallinity. This value is fairly small when compared to those for polyethylene $(66 \mathrm{cal} / \mathrm{g})$ and polyproplylene $(33 \mathrm{cal} / \mathrm{g})$, and nearly the same as those for polydecamethylene sebacate $(13.8 \mathrm{cal} / \mathrm{g})$ and polydecamethylene adipate $(13.4 \mathrm{cal} / \mathrm{g})$. (5) The temperature dependence of the storage modulus, $E^{\prime}$, may be estimated fairly well with a simple assumption that " $E$ ' depends on $\chi$ alone". The mechanical and thermal properties of this polymer indicate that they behave as random copolymers with crystallizable (1,2 unit with syndiotactic conformation) and noncrystallizable components. Polymers with high $\chi$ are composed of molecules with long crystallizable sequences.

KEY WORDS 1,2-Polybutadiene / Crystallinity / X-Ray / Melting Behavior / DSC / Viscoelasticity /
\end{abstract}

A number of samples of 1,2-polybutadiene with different microstructures were prepared by the polymerization method reported previously by Takeuchi, et al. ${ }^{1}$ The polymers are semicrystalline (below $46 \%$ in degree of crystallinity) thermoplastics with characteristic thermal properties. They have more than $90-\% \quad 1,2$ unit and syndiotactic configuration.

In the series of studies on the "Bulk properties of syndiotactic 1,2-polybutadiene", certain bulk properties of polymers will be discussed in relation to industrial processing technique, such as injection molding, the blow molding and the biaxial stretching. In such processes, the specimen is usually deformed mainly over a range of temperature covering the melting as well as the crystallization temperatures.

In this paper, some fundamental bulk properties of the polymer are discussed in relation to the degree of crystallinity for temperatures around the melting range of the polymer.

\section{EXPERIMENTAL AND DISCUSSION}

\section{Characterization of Samples}

Polybutadiene is composed of three isomeric units, cis-1,4, trans-1,4 and 1,2-vinyl. Their contents were estimated by Morero's method ${ }^{2}$ with the data from the infrared spectra. The results are listed in Table I. The method proposed by $\mathrm{Mochel}^{3}$ was used to estimate the tacticity of 1,2-polybutadiene samples. The results obtained are listed in the same table.

Although the methods herein adopted are not fully accepted as established, ${ }^{4}$ it may be said that 1,2-unit is always predominant in our samples and the amount of 1,4-trans is negligible. Further, the content of syndiotactic configuration increases with the content of 1,2-unit. Intrinsic viscosity, $[\eta]$ was measured at $25^{\circ} \mathrm{C}$ in 
Table I. Characteristic values for molecular chain conformation and degree of crystallinity

\begin{tabular}{|c|c|c|c|c|c|c|c|c|}
\hline \multirow{2}{*}{$\begin{array}{l}\text { Sample } \\
\text { code }\end{array}$} & \multirow{2}{*}{$\chi^{\mathrm{a}}, \%$} & \multicolumn{3}{|c|}{ Isomeric structure, $\%$} & \multicolumn{3}{|c|}{ Tacticity, $\%$} & \multirow{2}{*}[\eta]{, $\mathrm{d} l / \mathrm{g}$} \\
\hline & & 1,2 & $1,4-c i s$ & 1,4-trans & synd. & iso. & atac. & \\
\hline $\mathrm{S} 00$ & 0 & 82 & 18 & 0 & 23 & 36 & 41 & 2.20 \\
\hline S10 & 10 & & & & & & & 2.48 \\
\hline S15 & 15 & & & & & & & 1.38 \\
\hline S18 & 18 & 90 & 10 & 0 & 51 & 0 & 49 & 1.83 \\
\hline S25 & 25 & 92 & 8 & 0 & 66 & 0 & 34 & 1.24 \\
\hline S32 & 32 & 97 & 3 & 0 & 77 & 0 & 23 & 0.65 \\
\hline S42 & 42 & & & & & & & 1.20 \\
\hline S46 & 46 & & & & & & & 1.18 \\
\hline
\end{tabular}

a Degree of crystallinity at $30^{\circ} \mathrm{C}$ measured by density gradient tube.

toluene and listed in Table I.

For crystalline polymers, the degree of crystallinity is one of the most important factors affecting the mechanical properties of polymers. Mechanical properties are also largely affected by the thermal history of the specimen before measurement. Test specimens were prepared by the method discribed in the next paragraph and their densities measured by means of the density gradient tube technique at $30^{\circ} \mathrm{C}$. Denoting the weight fraction of the crystalline phase by $\chi$, it follows that

$$
\frac{1}{D}=\frac{\chi}{D_{\mathrm{c}}}+\frac{\chi-1}{D_{\mathrm{a}}}
$$

where $D$ is the density of a sample and the subscripts a and $\mathrm{c}$ stand for the amorphus and crystalline phases, respectively.

Natta and Corradini ${ }^{5}$ have presented a value of $0.963 \mathrm{~g} / \mathrm{cm}^{3}$ for $D_{\mathrm{c}}$ by an electron diffraction analysis. For $D_{\mathrm{a}}$, a value of $0.889 \mathrm{~g} / \mathrm{cm}^{3}$, the density of the sample $\mathrm{SOO}^{*}$, was used, because it appeared from its X-ray diffraction pattern to be noncrystalline. The real value of $D_{\mathrm{a}}$, however, may be slightly smaller than 0.889 , because a weak Debye-Scherrer ring was observed in the X-ray photograph and a small endothermic peak also observed. The value of $\chi$ determined by eq 1 , however, seems to have an error small enough to be neglected. The values of $\chi$ are listed in Table $\mathrm{I}$.

Another factor which characterizes the molecular chain conformation of the polymer is the sequence length distribution of the conformational component such a 1,2-unit with the syndiotactic conformation. No information on this factor is available.

Table I may indicate, however, that the 1,2 unit with syndiotactic conformation is the main component which affects the degree of crystallinity, since the other components such as the 1,2 unit with atactic conformation and the 1,4cis configuration are known to be apparently noncrystallizable over this temperature range. Further, the degree of crystallinity per weight fraction of the 1,2-unit with syndiotactic conformation are $0.39,0.41$ and 0.43 for $\mathrm{S} 18,{ }^{*} \mathrm{~S} 25^{*}$ and $\mathrm{S} 32$, respectively. This suggests that the degree of crystallinity increases linearly or with a slightly upward curvature with increasing weight fraction of this particular component.

\section{Preparation of Test Pieces}

Each sample was press-molded at a designated temperature under the following conditions; the periods for preheating, compression at $300 \mathrm{Kg} / \mathrm{cm}^{2}$ and cooling with water being $5 \mathrm{~min}, 10 \mathrm{~min}$ and $5 \mathrm{~min}$, respectively. The molding temperature was selected by taking account of the melting temperature and the melt viscosity of the sample. They were $160^{\circ} \mathrm{C}$ for S00, S10, S15, S18 and $\mathrm{S} 25,130^{\circ} \mathrm{C}$ for $\mathrm{S} 32$, and $200^{\circ} \mathrm{C}$ for $\mathrm{S} 42$ and $\mathrm{S} 46$, respectively.

Since differences is thermal history are known to have little effect on crystallization at temperatures above $130^{\circ} \mathrm{C}$, we may expect that, for the samples thus press-molded, they would be in nearly the same situation from the thermal

* The figures indicate the degree of crystallinity in percent. 


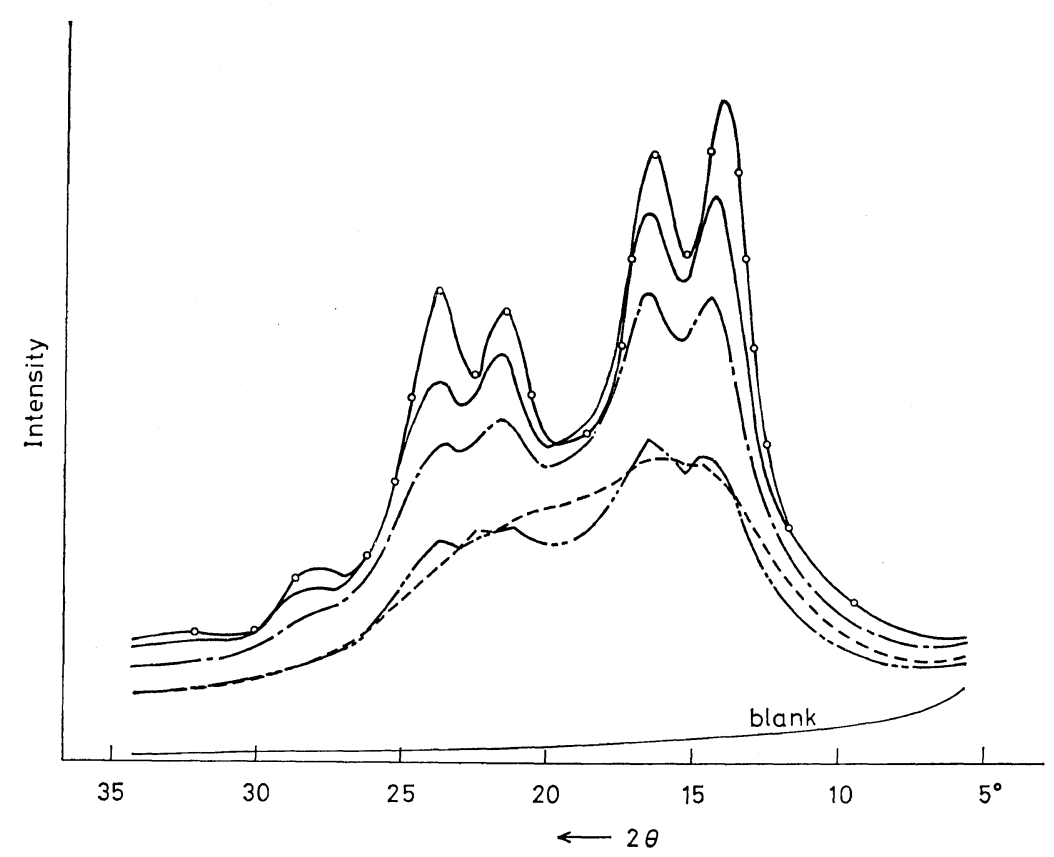

Figure 1. X-ray diffraction patterns of sample with different degree of crystallinity; S46 $\left(-\mathrm{O}^{-}\right), \mathrm{S} 32$ (-), S25 (- - ), S10 (-- ), S00 (-- -).

history point of view.

\section{Analysis of X-Ray Diffraction Peaks}

Figure 1 shows the scintillation counter registration curves for the transmission diffraction of a $\mathrm{Cu}-\mathrm{K} \alpha \mathrm{X}$-ray radiation measured at room temperature for the samples. Diffraction angles, $2 \theta$ at maximum intensity of S46 correspond to spacings of $6.42,5.49,4.13$ and $3.74 \AA$, respectively. The values are almost identical with those reported by Natta and Corradini. ${ }^{5}$ Such agreement leads to the conclusion that our specimens have the same lattice structure as that attributed by Natta, et al., to syndiotactic 1,2 -polybutadiene with a density of $0.92 \mathrm{~g} / \mathrm{cm}^{3}$.* Thus the structure of a unit cell is estimated to be orthorhombic with $a=10.98, b=6.60$ and $c($ molecular chain axis $)=5.14 \AA$.

Assignment of the diffraction peaks was made by comparing the inverse square of the space distance, $l / d^{2}$, observed with the one of $[h k l]$ plane calculated by the following relation.

$$
\frac{l}{d^{2}}=\frac{h^{2}}{a^{2}}+\frac{k^{2}}{b^{2}}+\frac{l^{2}}{c^{2}}
$$

* The density of $0.92 \mathrm{~g} / \mathrm{cm}^{3}$ corresponds to a degree of crystallinity of about $44 \%$ from eq 1 .
Table II. Assignment of diffraction peaks for sample S46

\begin{tabular}{cccc}
\hline $\begin{array}{c}\text { Peak } \\
\text { number }\end{array}$ & $\begin{array}{c}\text { Identified } \\
\text { surface }\end{array}$ & $1 / d^{2}$, obsd. & $1 / d^{2}$, calcd. \\
\hline P1 & 010 & 0.0243 & 0.0230 \\
P2 & 200 & 0.0332 & 0.0332 \\
P3 & 110 & & 0.0313 \\
& 210 & 0.0568 & 0.0561 \\
P4 & 011 & & 0.0608 \\
P5 & 111 & 0.0715 & 0.0691 \\
& 120 & 0.1001 & 0.1001 \\
\hline
\end{tabular}

Results are shown in Table II, designating the peaks P1, P2, P3, P4, and P5 in the order of increasing $2 \theta$.

In Figure 1, it can be observed that the diffraction angle $2 \theta$ of $\mathrm{P} 1$ corresponding to the (010) plane varies with $\chi$, while those of the other peaks do not. The values of $2 \theta$ (P1) of $\mathrm{S} 46, \mathrm{~S} 32$ and $\mathrm{S} 25$ are $13.8^{\circ}, 14.2^{\circ}$, and $14.5^{\circ}$, respectively. It seems to be inadequate to explain these results as the change of space distance of the (010) plane with the change in the degree of crystallinity. It would be better discussed in connection with the fact that the size 


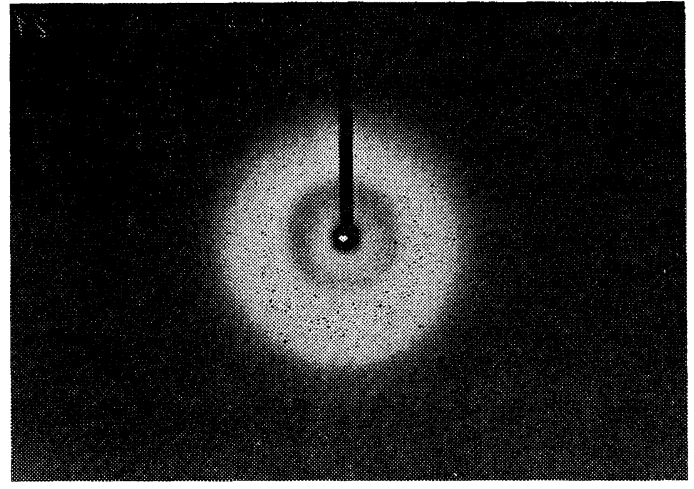

No. 1, SOO

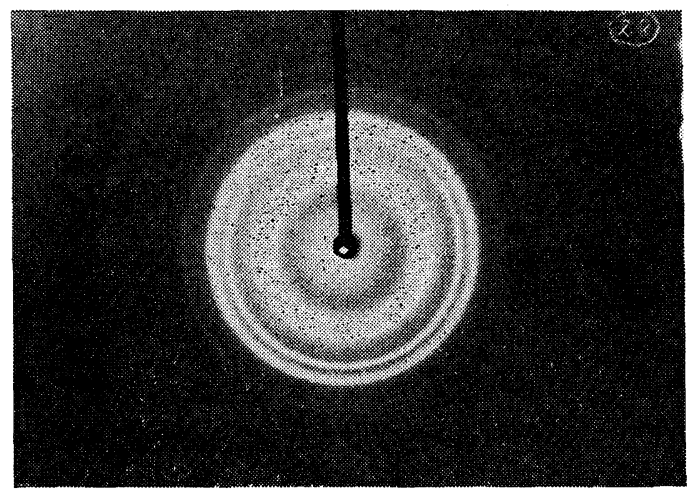

No. 3, S46

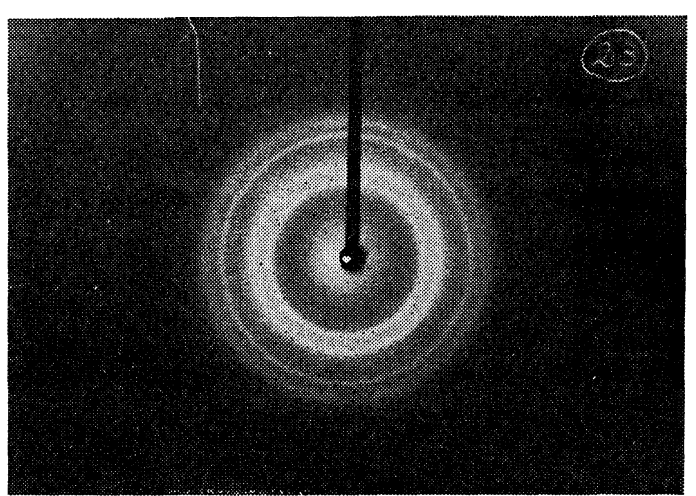

No. 2, S25

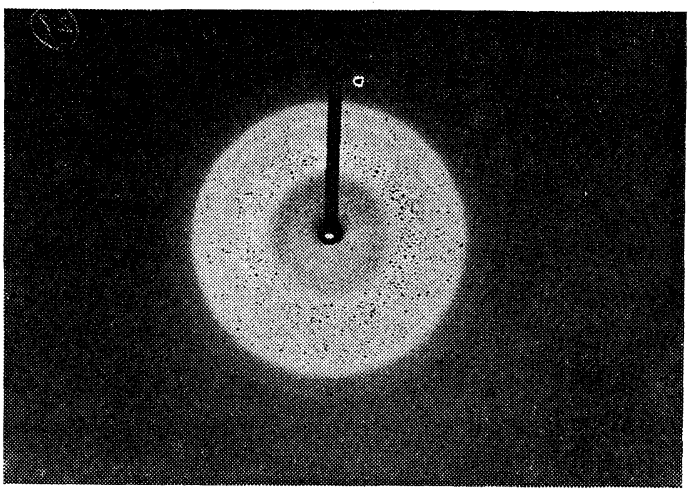

No. 4, S25

Figure 2. X-ray diffraction photographs of samples S00, S25, and S46 taken from through view and of S25 taken from edge view.

of the crystal is extremely small and the crystallite contains many defects due to the random distribution of noncrystallizable isomeric components. The size of the crystal has been estimated from the electron microscopic photographs. ${ }^{12}$ The defects contained in the crystallite will be discussed later in this paper.

Figure 2 shows X-ray photographs of the pressmolded specimens of S00, S25, and S46. The photographs No. 1, No. 2, and No. 3 in the figure were taken from a through view (where the incident beam of X-ray is perpendicular to the film surface). As seen in Figure 1, each diffraction ring becomes sharper with increasing degree of crystallinity.

In the photograph of S00, an amorphous hallow is predominant suggesting that the sample SOO is noncrystalline, though some weak rings due to crystalline diffraction are also observed.

The photograph No. 4 for S25 was taken from an edge view (the incident beam is parallel to the film surface) to compare it with the photograph No. 2 (through view). It is found that the orientation of the crystals is random in the press-molded specimen.

Temperature Dependence of Degree of Crystallinity

The degree of crystallinity in samples is expected to vary with temperature and it was estimated by analysis of the X-ray diffraction intensity distribution scanned at respective temperatures. A sketch of the specimen holder consisting of a plate of Bakelite and thin polyester film is shown in Figure 3.

The specimens were measured along $2 \theta$ at a scanning rate of $2 \% / \mathrm{min}$ after annealing for 10 min at given temperatures in a thermo-controlled chamber. Typical diffraction curves at 27.5, 70 and $100^{\circ} \mathrm{C}$ are shown in Figure 4. In the figure it is seen that the degree of crystallinity decreases with increasing temperature. No 
Bulk Properties of Syndiotactic 1,2-Polybutadiene. I.

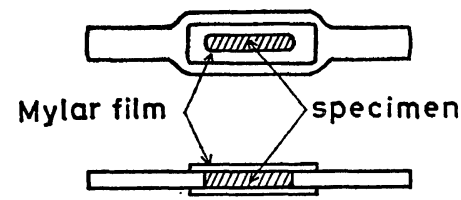

Figure 3. Schematic diagram of a specimen and its holder used to measure the $\mathrm{X}$-ray diffraction pattern at temperatures above $30^{\circ} \mathrm{C}$. definite method has been established as yet to divide the $\mathrm{X}$-ray diffraction intensity distribution into two parts, namely one due to the crystalline and the other due to the amorphous contributions. Usually a curve is drawn like the dotted curve in Figure 5, but naturally it is ambiguous. In this paper, only a limited range of the diffraction intensity curve was used in a range of $2 \theta$ between $19.6^{\circ}$ and $27^{\circ}$, and a

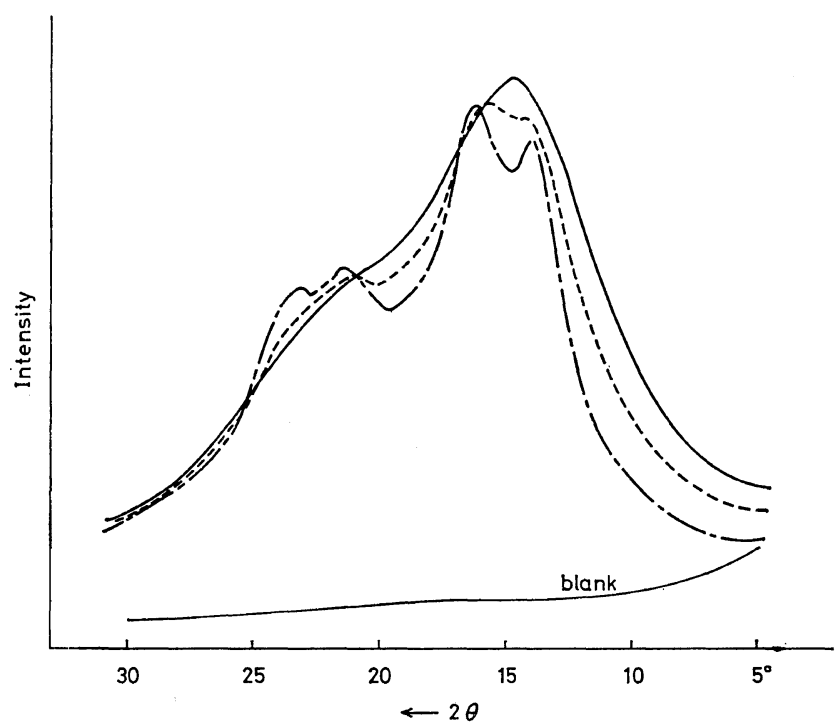

Figure 4. Diffraction patterns of a sample $\mathrm{S} 25$ measured at $27.5^{\circ} \mathrm{C}(--), 70^{\circ} \mathrm{C}(---)$, and $100^{\circ} \mathrm{C}(-)$.

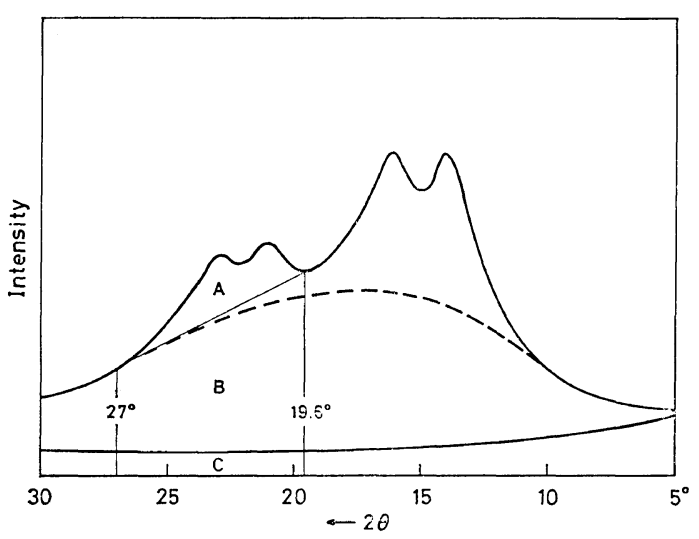

Figure 5. Schematic representation by drawing a base line which may be expected to due to the amorphous contribution (-- ) and a straight line drawn for convenience to derive the contribution. straight line was drawn as shown in Figure 5 . In the figure, the areas $A, B$, and $C$ are assumed to be due to the crystalline, amorphous and blank, respectively. Putting $A$ and $B$ as a function of temperature, $\chi(T)$ is defined by the following equations.

$$
\begin{aligned}
& \frac{A(T)}{A(T)+B(T)} \equiv R(T) \\
& \chi(T)=\frac{R(T)}{R\left(T_{\mathrm{r}}\right)} \chi\left(T_{\mathrm{r}}\right) .
\end{aligned}
$$

Here $\chi\left(T_{\mathrm{r}}\right)$ is the degree of crystallinity estimated from the density at a reference temperature $\left(T_{\mathrm{r}}\right)$ of $30^{\circ} \mathrm{C}$. Values of $\chi(T)$ of samples $\mathrm{S} 25, \mathrm{~S} 32$, and $\mathrm{S} 46$ are shown as a function of temperature in Figure 6. From the figure, it is found that the degree of crystallinity 


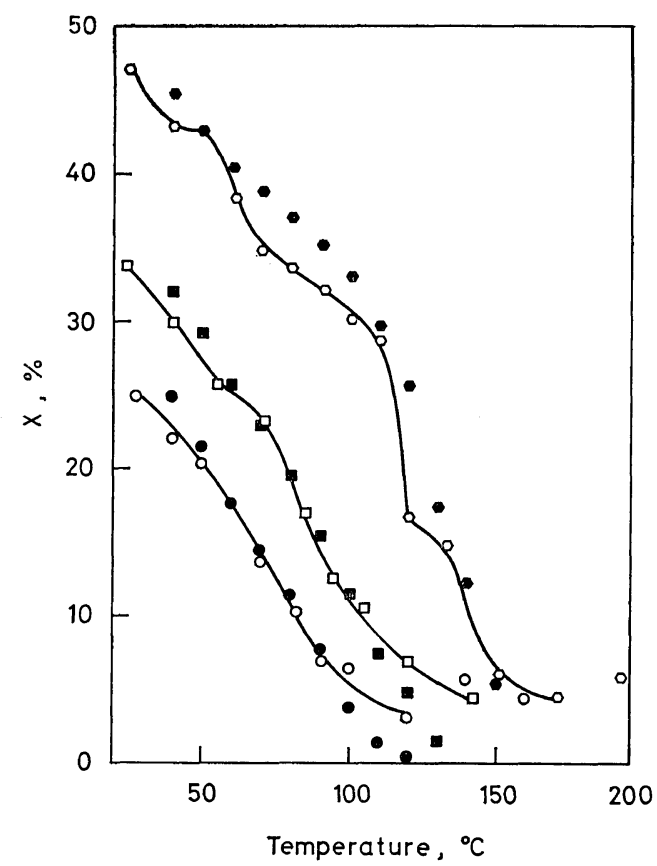

Figure 6. Temperature dependence of the degree of crystallinity; from X-ray, S46 (O), S32 ( $\square$ ),

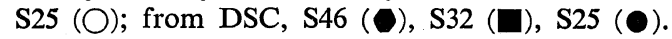

decreases almost linearly with increasing temperature.

\section{Thermal Analysis by Means of DSC}

It is well known that the DSC thermogram of a crystalline polymer is largely affected by the heating rate as well as the thermal history of the sample. The main purpose of this paragraph is to characterise the thermal properties of this polymer in relation to its degree of crystallinity. In this experiment, the rate of heating was set at $20^{\circ} \mathrm{C} / \mathrm{min}$, and the effects of superheating were not very large at this heating rate.

Experiments were planned for the following points; (1) the dependence of both $T_{\mathrm{g}}$ and temperature range of fusion on the degree of crystallinity, (2) the specific heat of fusion of the crystal and (3) the temperature dependence of the degree of crystallinity in a DSC thermogram. Measurements were performed by using the Thermoflex (Type 8001) manufactured by Rigaku Denki Co. The press-molded specimen of 15 to $20 \mathrm{mg}$ sealed within an aluminum pan was cooled previously from room temperature to

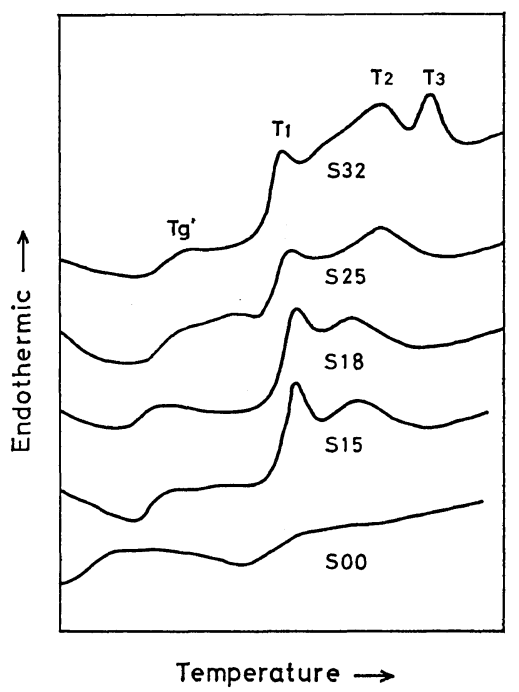

Figure 7. A series of DSC thermograms of the samples scanned at constant heating rate $20^{\circ} \mathrm{C} / \mathrm{min}$.

$-78^{\circ} \mathrm{C}$ at a cooling rate of about $40^{\circ} \mathrm{C} / \mathrm{min}$ and then heated at the heating rate of $20^{\circ} \mathrm{C} / \mathrm{min}$, while recording the differential power input.

Figure 7 shows typical DSC thermograms of the samples with different degrees of crystallinity. It is seen from the figure that the fusion of the crystal occurs over a very wide range of temperature, and that the thermogram changes systematically with the degree of crystallinity. For the fusion of crystalline polymers, either the temperature at the peak of the thermogram or the temperature at the terminated point of the fusion is generally used as a measure of the melting temperature. Certain characteristic temperatures such as an apparent glass transition temperature $T_{\mathrm{g}}{ }^{\prime}$, temperatures at the endothermic peaks $T_{1}, T_{2}$, and $T_{3}$ and also the temperature at the terminated point of the fusion $T_{e}$ are pointed out in Figure 8. The values of $T_{1}, T_{2}$, and $T_{\mathrm{e}}$ of the samples are listed in Table III. From the table, it was found that $T_{2}$ and $T_{\mathrm{e}}$ increase with the degree of crystallinity, but $T_{1}$ remains practically unchanged. The value of $T_{\mathrm{e}}$ is plotted as a function of the degree of crystallinity $\chi$ in Figure 9 , showing that $T_{\mathrm{e}}$ changes almost linearly with $\chi$.

It should be remembered that $T_{\theta}$ thus obtained depends on the heating rate. As an example, it was observed that $T_{\mathrm{e}}$ of S25 decreases about 
Bulk Properties of Syndiotactic 1,2-Polybutadiene. I.

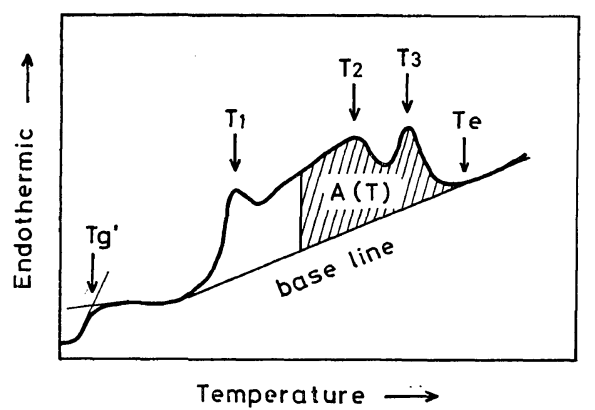

Figure 8. A Schematic representation of a method determining an apparent glass transition temperature $T_{\mathrm{g}}$, melting temperatures $T_{1}, T_{2}, T_{3}$ and temperature at the termination of the melting $T_{\mathrm{e}}$.

Table III. Characteristic temperatures from DSC thermograms

\begin{tabular}{ccccc}
\hline Sample & $T_{\mathrm{g}},{ }^{\circ} \mathrm{C}$ & $T_{1},{ }^{\circ} \mathrm{C}$ & $T_{2},{ }^{\circ} \mathrm{C}$ & $T_{\mathrm{e}},{ }^{\circ} \mathrm{C}$ \\
\hline S00 & -38 & & 61 & \\
S10 & -18 & 51 & & 96 \\
S15 & -21 & 48 & 75 & 112 \\
S18 & -24 & 49 & 76 & 116 \\
S25 & -19 & 48 & 93 & 124 \\
S32 & -14 & 47 & 101 & 148 \\
S42 & 5 & 50 & 144 & 165 \\
S46 & 7 & 48 & 154 & 177 \\
\hline
\end{tabular}

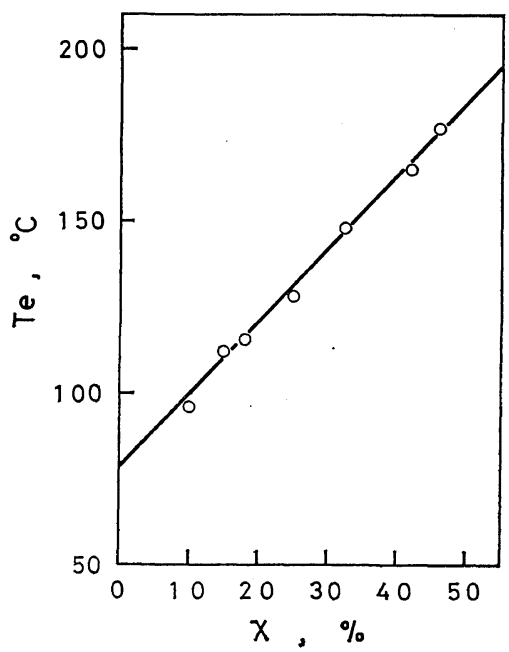

Figure 9. A relation between the $T_{\mathrm{e}}$ and $\chi$ at $30^{\circ} \mathrm{C}$.

$10^{\circ} \mathrm{C}$ when the heating rate changes from $20^{\circ} \mathrm{C}$ to $4^{\circ} \mathrm{C} / \mathrm{min}$.

The Heat of fusion of the crystallite was

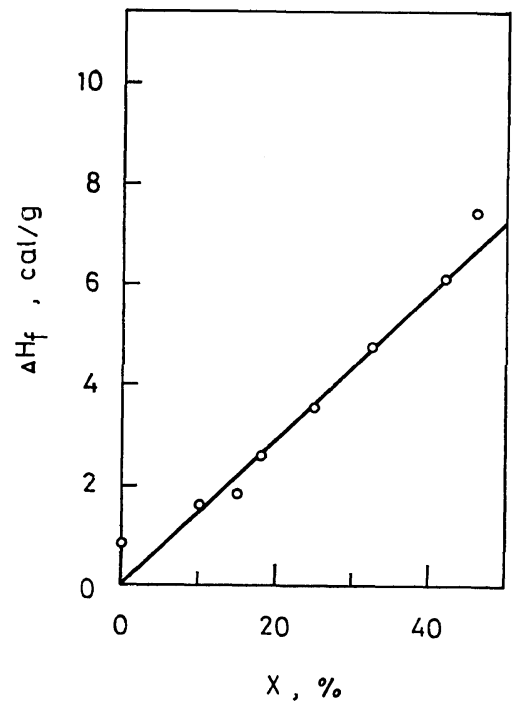

Figure 10. Specific heat of fusion $\Delta H_{\mathrm{f}}$ as a function of $\chi$.

obtained by integrating the endothermic peaks as illustrated in Figure 8, comparing the area with that of a known weight of reference sample Indium $(6.81 \mathrm{cal} / \mathrm{g})$. The heat of fusion per gram of the sample $\left(\Delta H_{\mathrm{f}}\right)$ is shown as a function of the degree of crystallinity in Figure 10. A linear relation between $\Delta H_{\mathrm{f}}$ and $\chi$ seems to exist as shown in the figure. An extrapolation gives $14.5 \mathrm{cal} / \mathrm{g}$ for $100-\%$ crystallinity. Although the extrapolation was made by using data for a relatively low degree of crystallinity (below $46 \%$ ), it is very small as compared to those of polyethylene $(66 \mathrm{cal} / \mathrm{g})^{7}$ and polypropylene (33 $\mathrm{cal} / \mathrm{g})^{8}$ and is nearly the same as those of polydecamethylene adipate $(13.4 \mathrm{cal} / \mathrm{g})^{9}$ and polydecamethylene sebacate $(13.8 \mathrm{cal} / \mathrm{g}){ }^{9}$

Since the polmer molecules are composed of crystallizable (1,2-unit with syndiotactic conformation) and noncrystallizable sequences, the sequence length and its distribution may be different for individual molecules. Block copolymers with a long sequence length, which is one of the extremes, lead to a high crystallinity and to a high melting temperature. Random copolymers with a very short sequence length (the other extreme), form small and imperfect crystallites to give lower melting temperatures. The broad melting temperature range may indicate that the sample is a mixture of 
molecules with a variety of sequence lengths and distributions.

$T_{\mathrm{g}}{ }^{\prime}$ evaluated from the DSC thermogram, which is an apparent value of $T_{\mathrm{g}}$, is usually slightly different from $T_{\mathrm{g}}$ at equilibrium. $T_{\mathrm{g}}{ }^{\prime}$ in Figure 8 can be converted to $T_{\mathrm{g}}$ by

$$
T_{\mathrm{g}}=1.05 T_{\mathrm{g}}^{\prime}-12 \text {. }
$$

Equation 4 is the calibrated relationship* between the values of $T_{\mathrm{g}}$ in literature ${ }^{6}$ and those observed for $T_{\mathrm{g}}{ }^{\prime}$ for various materials. The values of $T_{\mathrm{g}}$ thus obtained are also listed in Table III.

Temperature dependence of the degree of cyrstallinity may be measured with a DSC thermogram when the heating rate is sufficiently small. $\chi(T)$ was calculated by eq 5

$$
\chi(T)=\chi\left(T_{\mathrm{r}}\right) \frac{A(T)}{A\left(T_{\mathrm{r}}\right)}
$$

where $A(T)$ is the hatched area shown in Figure 8 and $T_{\mathrm{r}}$ is the reference temperature $30^{\circ} \mathrm{C}$. The results are illustrated in Figure 6 compared with those obtained via the X-ray diffraction method in a sort of static manner. The data from both the X-ray diffraction and DSC methods show fairly good agreement with each other. Although some questions may arise in the accuracy of the value of $\chi$ obtained by the methods mentioned above, the data may be regarded as those at a quasi-equiliblium state. The results shown in Figure 6 are reliable enough to draw the following qualitative conclusions and the fact that the samples have a broad range of melting temperature and that the melting temperatures depends largely on the degree of crystallinity (Figure 9) indicate that they are qualitatively identical with the results predicted by Flory ${ }^{10}$ for copolymers consisting of crystallizable and noncrystallizable units.

\section{Dynamic Measurement}

To investigate the relationship between mechanical properties and the degree of crystallinity, dynamic Young's modulus $E^{\prime}$ and $\tan \delta$ were measured at temperatures ranging from $-50^{\circ}$ to $130^{\circ} \mathrm{C}$ at a fixed frequency of $10 \mathrm{~Hz}$. Test specimens were cut out of the press-molded films prepared by the method described in the previous paragraph and measured using a direct-

\footnotetext{
* Unpublished work by one of the authors (C.T.).
}

reading dynamic mechanical spectrometer. The measurements were performed from lower to higher temperatures in a stepwise manner. Figures 11 and 12 shows the temperature dependence of $E^{\prime}$ and $\tan \delta$, respectively, for all specimens.

In the figures, the temperature dependence of $E^{\prime}$ and $\tan \delta$ changes gradually from, those typical of an amorphous polymer to those of a semicrystalline polymer as the degree of crystallinity increases. In order to investigate how $E^{\prime}$ depends on $\chi, E^{\prime}$ at $30^{\circ} \mathrm{C}$ was replotted in Figure 13 as a function of $\chi$ at $30^{\circ} \mathrm{C}$. At this temperature, the values of $\tan \delta$ of the specimens are nearly the same (Figure 12) and any melting of the crystal was not observed in the DSC thermogram. The figure shows that $E^{\prime}$ increases exponentially with $\chi$ and it is approximately expressed by

$$
\begin{aligned}
E^{\prime} & \doteqdot E_{0}{ }^{\prime} \exp (k \chi) \\
& =E_{0}{ }^{\prime}\left(1+11.2 \chi+62.7 \chi^{2}+\cdots\right)
\end{aligned}
$$

This indicates that the dependence of $E^{\prime}$ on $\chi$ is much stronger than that in filled rubber

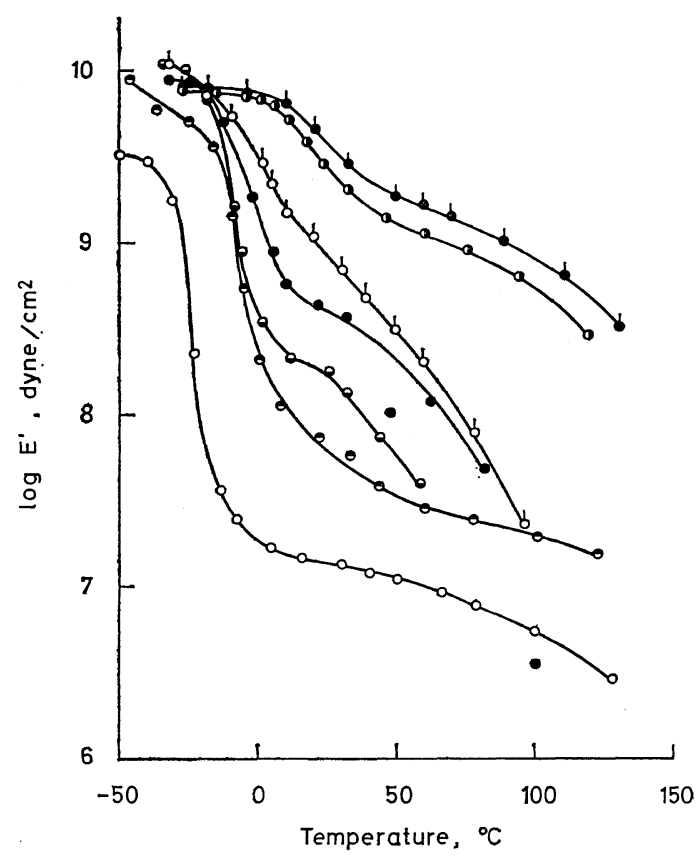

Figure 11. Temperature dispersion of dynamic Young's modulus $E^{\prime}$ of samples $\mathrm{S} 00(\mathrm{O}), \mathrm{S} 10(\boldsymbol{\theta})$,

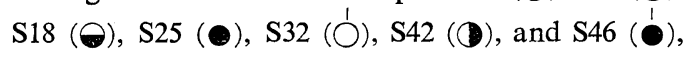
measured at $10 \mathrm{~Hz}$. 
Bulk Properties of Syndiotactic 1,2-Polybutadiene. I.

on volume fraction of filler $\phi$, which was derived by Guth, et al. ${ }^{10}$ That is,

$$
E=E_{0}\left(1+2.5 \phi+14.1 \phi^{2}+\cdots\right) \text {. }
$$

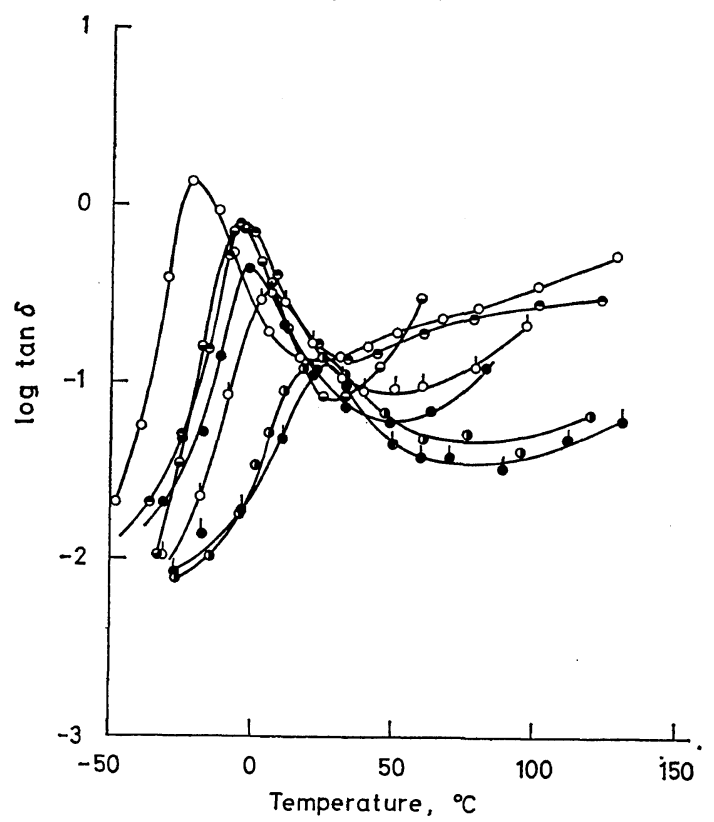

Figure 12. Temperature dispersion of $\tan \delta$ of the

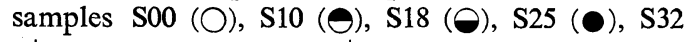
(ல), $\mathrm{S} 42(\mathrm{O})$, and $\mathrm{S} 46$ (') measured at $10 \mathrm{~Hz}$.

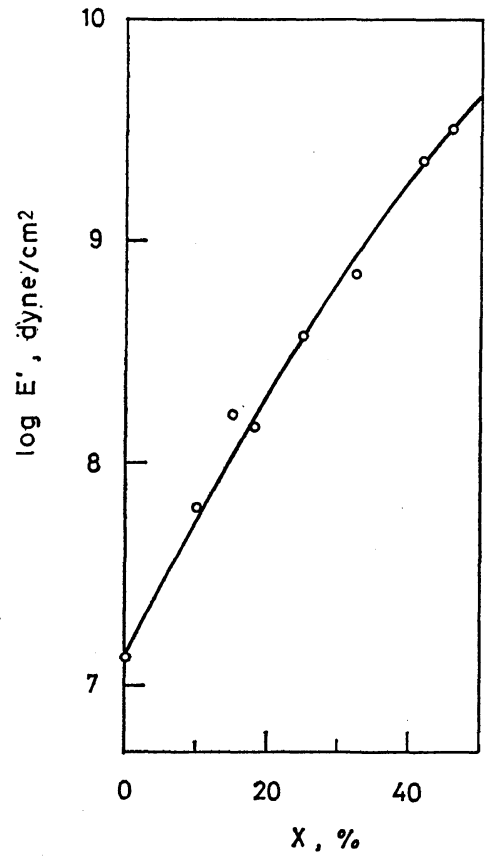

Figure 13. A relation between $E^{\prime}$ and $\chi$ at $30^{\circ} \mathrm{C}$.
For the description of the viscoelasticity of semicrystalline polymer whose degree of crystallinity varies with temperature, Ninomiya, et al., presented a phenomenological theory and examined the validity for a gell of Cellulose Nitrate. ${ }^{11}$ Some investigations for viscoelasticity of the polymer including both temperature and frequency dependences will be reported in a succeeding paper of this series.

In this paper attention was directed towards the temperature dependence of $E^{\prime}$ in relation to the temperature dependence of $\chi(T)$ as shown in Figure 6. As an approximation, we assumed that " $E$ ' could be directly derived from $\chi(T)$ alone", neglecting the temperature dependence of viscoelasticity in the amorphous phase as well as the interaction force between the crystal and the amorphous phases. $E^{\prime}(T)$ was then formulated from two relationships; the one between $E^{\prime}\left(30^{\circ} \mathrm{C}\right)$ and $\chi\left(30^{\circ} \mathrm{C}\right)$ which is shown in Figure 13 and the other between $\chi$ and $T$ obtained from the X-ray diffraction method shown in Figure 6. The estimated values of $E^{\prime}(T)$ of sample S25, S32, and S46 are shown in full lines together with $E^{\prime}(T)$ observed (circles) in Figure 14. The observed and estimated values of $E^{\prime}(T)$ show fairly good agreement taking the roughness in the assumptions proposed into account. For $\mathrm{S} 32$, the estimated values of $E^{\prime}(T)$,

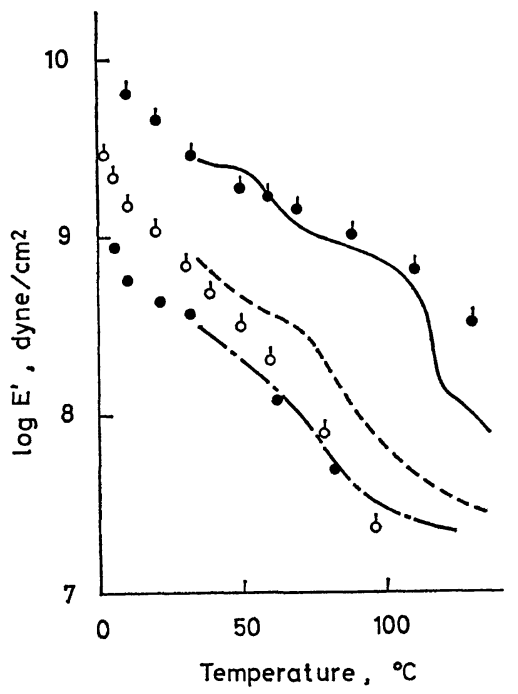

Figure 14. Estimated and measured values of $E^{\prime}$. The estimation was made using the temperature dependence of $\chi$ by X-ray. 
however, are larger than the observed ones. This may be related to the lower intrinsic viscosity of $\mathrm{S} 32$ as shown in Table $\mathrm{I}$.

Acknowledgement. The authors wish to express their sincere thanks to Mr. Chikara Honma, Misses Fusako Shimizu and Yoshiko Akiyama for technical assistance in carring out the experiments and calculations. The authors also acknowledge the helpful comments made by Drs. T. Honma, M. Ninomi and K. Ninomiya, and also the invaluable discussions by Drs. $M$. Abe, K. Yagii and N. Shiraishi of Development Laboratory of our company.

\section{REFERENCES}

1. Y. Takeuchi, M. Ichikawa, and K. Mori, Paper presented at 5th Polymer Symposium, Osaka, Japan, 1965.
2. D. Morero, A. Santambrogio, L. Porri, and F. Ciampelli, Chem. e. Ind., 41, 758 (1959).

3. V. D. Mochel, J. Polym. Sci., Part A-1, 10, 1009 (1972).

4. Y. Tanaka and Y. Takeuchi, ibid., Part A-2, 9, 43 (1971).

5. G. Natta and P. Corradini, ibid., 20, 251 (1956).

6. Polymer Handbook, J. Brandrup, E. H. Imergut, John Wiley \& Sons, Inc., New York, N.Y., 1966.

7. A. Peterlin and G. Meinel, J. Appl. Phys., 36, 3028 (1965).

8. K. Kamide and K. Nakamura, Seni Gakukai Shi (J. Soc. Fiber Sci. Technol.), 24, 486 (1968).

9. R. D. Evans, H. R. Mighton, and P. J. Flory, J. Amer. Chem. Soc., 72, 2018 (1951).

10. P. J. Flory, Trans. Faraday Soc., 51, 848 (1955).

11. K. Ninomiya and J. D. Ferry, J. Polym. Sci., 5, 195 (1967).

12. M. Niinomi, T. Katsuta, and Y. Obata, Rep. Progr. Polym. Phys. Japan, 17, 243 (1974). 